\title{
OPEN High-isolation antenna array using SIW and realized with a graphene layer for sub-terahertz wireless applications
}

Mohammad Alibakhshikenari ${ }^{\boxplus}$, Bal S. Virdee ${ }^{2}$, Shahram Salekzamankhani ${ }^{2}$, Sonia Aïssa ${ }^{3}$ Chan H. See ${ }^{4}$, Navneet Soin ${ }^{5}$, Sam J. Fishlock ${ }^{5}$, Ayman A. Althuwayb ${ }^{6}$, Raed Abd-Alhameed ${ }^{7}$, Isabelle Huynen ${ }^{8}$, James A. McLaughlin ${ }^{5}$, Francisco Falcone ${ }^{9,10}$ \& Ernesto Limiti ${ }^{1}$

This paper presents the results of a study on developing an effective technique to increase the performance characteristics of antenna arrays for sub-THz integrated circuit applications. This is essential to compensate the limited power available from sub-THz sources. Although conventional array structures can provide a solution to enhance the radiation-gain performance however in the case of small-sized array structures the radiation properties can be adversely affected by mutual coupling that exists between the radiating elements. It is demonstrated here the effectiveness of using SIW technology to suppress surface wave propagations and near field mutual coupling effects. Prototype of $2 \times 3$ antenna arrays were designed and constructed on a polyimide dielectric substrate with thickness of $125 \mu \mathrm{m}$ for operation across $0.19-0.20 \mathrm{THz}$. The dimensions of the array were $20 \times 13.5 \times 0.125 \mathrm{~mm}^{3}$. Metallization of the antenna was coated with $500 \mathrm{~nm}$ layer of Graphene. With the proposed technique the isolation between the radiating elements was improved on average by $22.5 \mathrm{~dB}$ compared to a reference array antenna with no SIW isolation. The performance of the array was enhanced by transforming the patch to exhibit metamaterial characteristics. This was achieved by embedding the patch antennas in the array with sub-wavelength slots. Compared to the reference array the metamaterial inspired structure exhibits improvement in isolation, radiation gain and efficiency on average by $28 \mathrm{~dB}, 6.3 \mathrm{dBi}$, and 34\%, respectively. These results show the viability of proposed approach in developing antenna arrays for application in sub-THz integrated circuits.

Wireless communication bands are increasingly becoming highly crowded and the ever-increasing demand for more bandwidth has provoked the exploitation of the yet unexplored electromagnetic spectrum in the subterahertz $(\mathrm{THz})$ region $^{1}$. Higher bandwidth afforded by the sub-terahertz band can achieve extremely high data-rates in the order of $0.1 \mathrm{THz} / \mathrm{s}$ for future wireless systems ${ }^{2}$. However, sub-terahertz radiation is absorbed by most materials, including the molecules in the atmosphere such as water vapor. For such systems, line-of-sight propagation is needed and even for such conditions, the range is rather limited due to the inherently high pathloss. Since path-loss is relatively high and the current sub- $\mathrm{THz}$ receivers are not sensitive, hence much effort needs to be devoted on maximizing both the radiation-gain and efficiency of sub-terahertz antennas.

Owing to the lack of sub-terahertz sources and detectors, this area of the electromagnetic spectrum has not been fully explored for wireless communication applications ${ }^{3}$. The up and down-converters as well as low-gain

${ }^{1}$ Electronic Engineering Department, University of Rome "Tor Vergata", Via del Politecnico 1, 00133 Rome, Italy. ${ }^{2}$ Center for Communications Technology \& Mathematics, School of Computing and Digital Media, London Metropolitan University, London N7 8DB, UK. ${ }^{3}$ Institut National de La Recherche Scientifique (INRS), University of Quebec, Montréal, OC H5A 1K6, Canada. ${ }^{4}$ School of Engineering and the Built Environment, Edinburgh Napier University, Edinburgh, UK. ${ }^{5}$ School of Engineering, Ulster University, Newtownabbey, Belfast, Northern Ireland BT37 0OB, UK. ' ${ }^{6}$ lectrical Engineering Department, Jouf University, Sakaka 72388, Saudi Arabia. ${ }^{7}$ Faculty of Engineering and Informatics, University of Bradford, Bradford, UK. ${ }^{8}$ Institute of Information and Communication Technologies, Electronics and Applied Mathematics, Université Catholique de Louvain, Ottignies-Louvain-la-Neuve, Belgium. ${ }^{9}$ Electric, Electronic and Communication Engineering Department, Public University of Navarre, 31006 Pamplona, Spain. ${ }^{10}$ Institute of Smart Cities, Public University of Navarre, 31006 Pamplona, Spain. ${ }^{\varpi}$ email: alibakhshikenari@ing.uniroma2.it 


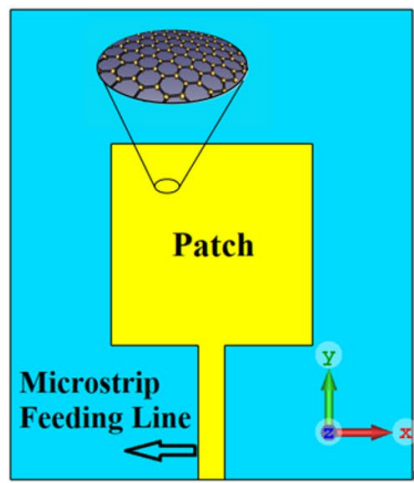

(a) Top view

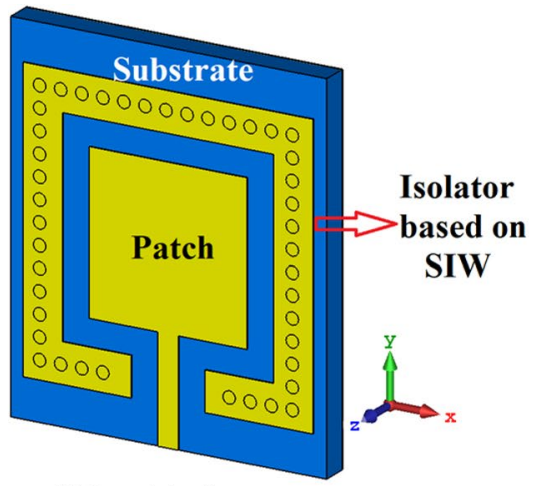

(b) Isometric view

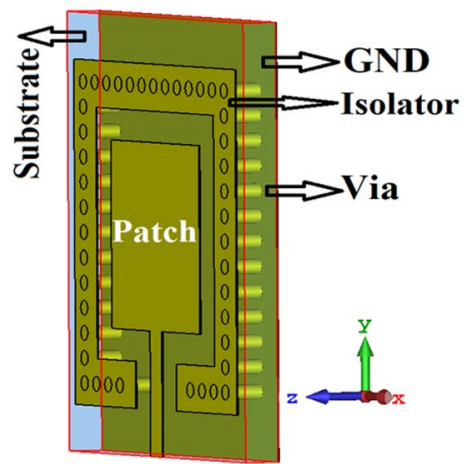

(c) Schematic view

Figure 1. Antenna structure: (a) reference antenna with no SIW technology, (b) and (c) the proposed antenna with SIW technology.

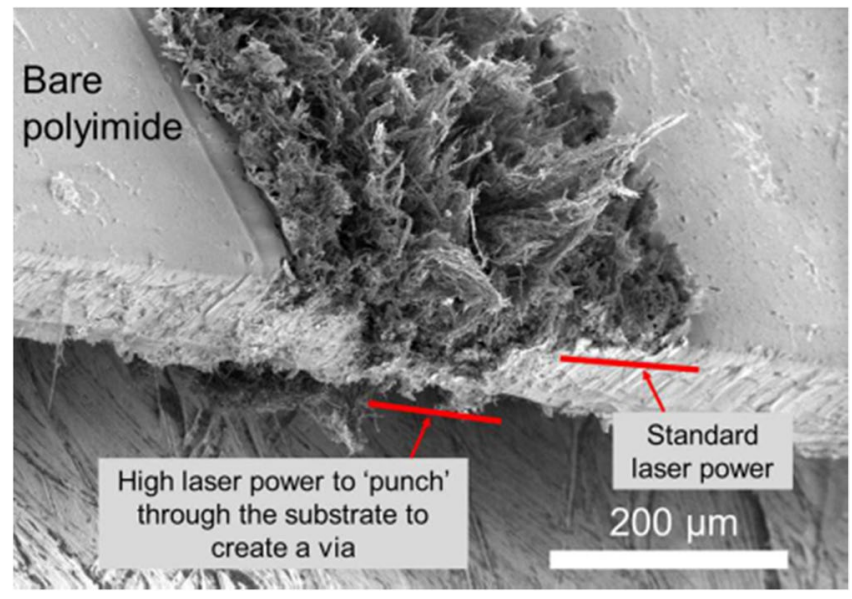

Figure 2. Scanning electron microscope (SEM) image of polyimide substrate ( $65^{\circ}$ tilt angle) to highlight how the vias are fabricated by increasing the scribing laser power to 'punch' through the substrate and create an electrically conducting through-connection.

amplifiers constituting the RF front-end of sub-terahertz communication system have restricted output power and bandwidth. Existing technology relies on mixing optical sources essentially lasers to generate low to moderate power-levels at sub-THz band. Receivers at such frequencies rely on direct detection using Schottky-diodes, which is not band selective and has a low dynamic range and sensitivity ${ }^{4}$.

At sub-THz frequencies metals exhibit a lower conductivity than at DC. This phenomenon enhances field infiltration within the metal at sub- $\mathrm{THz}$ and adversely affect the radiation efficiency of metallic antennas ${ }^{5}$. In addition to the low conductivity of metal at these frequencies, the effect of small geometric parameters of metallic antennas, specifically the width or radius of metallic traces which are smaller than 0.1 micron, must be considered. This is because numerical analysis reveals that small scale antennas of sub-100 nano-meters compared to their millimeter-sized counterparts results in significantly low radiation efficiency at sub-THz frequency due to the high surface resistance of metallic traces ${ }^{6}$. Moreover, because of the method employed for conductor deposition in the fabrication of ultra-thin metallic traces of sub-nano-meter thickness, the magnitude of the metal's conductivity becomes considerably lower than that of the bulk material. The low conductivity is attributed to the following factors: grain boundary scattering, surface scattering, and surface roughness ${ }^{7,8}$. These factors can considerably reduce the theoretically predicted radiation efficiency of antennas at sub-terahertz frequencies.

Recently various sub- $\mathrm{THz}$ antenna arrays have been investigated for $\mathrm{THz}$ spectroscopy based on slots ${ }^{9}$, printed dipoles ${ }^{10}$ or patch antennas ${ }^{11}$ however the radiation characteristics of these antennas are limited for practical applications. Hence there is demand for antennas at these frequencies with improved radiation gain and efficiency characteristics. Although high performance wideband horn antennas are now available ${ }^{12}$, they are bulky in structure even at very high frequencies. Instead, low profile planar antennas are desirable for ease of integration with RF-circuits to realize compact transceivers ${ }^{13,14}$. 


\begin{tabular}{|l|l|}
\hline Parameter & Dimension $(\mathbf{m m})$ \\
\hline Patch length & 3 \\
\hline Patch width & 3 \\
\hline Feedline length & 2 \\
\hline Feedline width & 0.4 \\
\hline SIW isolator width & 0.75 \\
\hline Gap between patch and isolator & 0.5 \\
\hline Gap between feedline and isolator & 0.5 \\
\hline Diameter of vias & 0.25 \\
\hline Gap between vias & 0.20 \\
\hline Thickness of graphene layer & 0.0005 \\
\hline Substrate thickness & 0.125 \\
\hline
\end{tabular}

Table 1. Dimensions of the antenna.

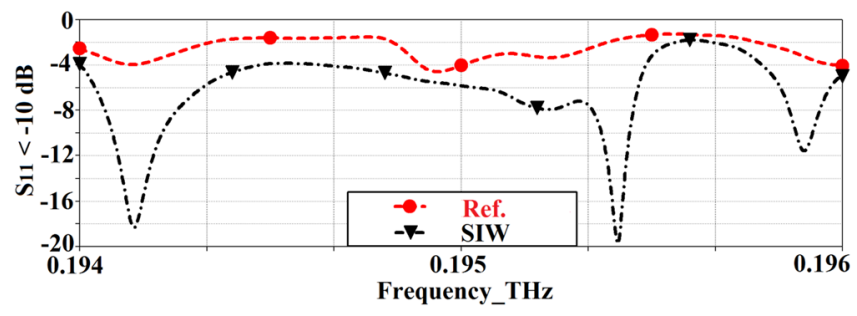

Figure 3. Simulated reflection-coefficient response of the reference and SIW-loaded patch antennas.

In this study the isolation, radiation-gain and efficiency of sub-terahertz array structure is improved by employing a combination of substrate integrated waveguide (SIW) and metamaterial (MTM) inspired technologies. The prototype antenna arrays were designed and analysed using numerical simulation tools, and the optimized designs fabricated and measured. The array structures were coated with a thin layer of Graphene as it supports the propagation of surface plasmon polaritons enabling it to operate in the sub-terahertz frequency band ${ }^{15,16}$. In ${ }^{17}$ it has been shown that plasmons in graphene layer strongly confine electromagnetic energy, and $i^{18}$ it has been shown that the application of a voltage to the graphene layer affects the phase characteristics of the antenna. These results show Graphene material opens exciting prospects for antenna development in the terahertz frequency band.

\section{Sub-terahertz antenna based on SIW Technology}

Geometries of the standard reference patch and the proposed SIW-loaded patch antenna are shown in Fig. 1. The patch antenna was constructed on a polyimide dielectric substrate (Kaneka, Apical NP, USA) with thickness of $125 \mu \mathrm{m}$, dielectric constant $\left(\varepsilon_{r}\right)$ of 3.5 , and loss-tangent $(\operatorname{tang} \delta)$ of 0.0027 . To produce the graphene patch, a CO2 laser (Universal Laser $230 \mathrm{VLS}$ ) was used to scribe the front and rear polyimide surfaces and thereby convert this into a graphene film, using the same experimental conditions as discussed in ${ }^{19}$. To produce the vias, the laser power was increased from $8.1 \mathrm{~W}$ (as used for the standard patch on the front face) to $15 \mathrm{~W}$, and the laser scribe was repeated twice in these areas, in order to 'punch' through the polyimide surface. A cross section of the via taken using an SEM (Hitachi SU5000 field emission scanning electron microscope) can be observed in Fig. 2. The proposed antenna includes SIW isolator which is framed around the square patch. Dimensions of the antenna are given in Table 1 . Reflection-coefficient response for both antennas over $0.194-0.196 \mathrm{THz}$ is shown in Fig. 3. The results reveal SIW isolation improves the impedance match of the antenna, which is pronounced at the resonance frequencies of $0.1942 \mathrm{THz}$ and $0.1954 \mathrm{THz}$. Surface current density distribution over the two antennas at their dominant resonant modes in Fig. 4 show the effectiveness of the SIW isolator in suppressing the propagating surface waves over the structure. Suppression of surface waves is desirable in antenna arrays to mitigate mutual coupling between adjacent radiating elements and thereby improve the antenna's radiation characteristics. Radiation gain and efficiency plots of both antennas at the resonant frequencies in Fig. 5 show the SIW isolator significantly enhances the radiation-gain and efficiency properties over the reference antenna, due to reduction in substrate loss. 


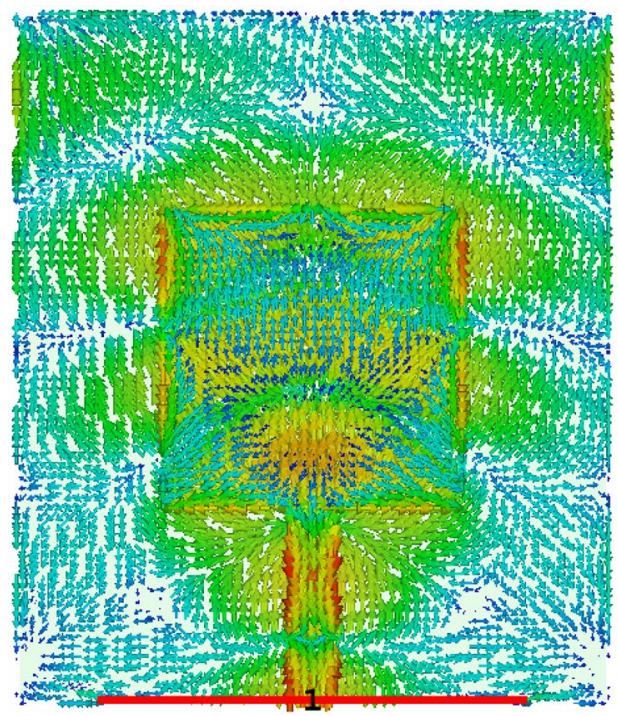

With no SIW isolator

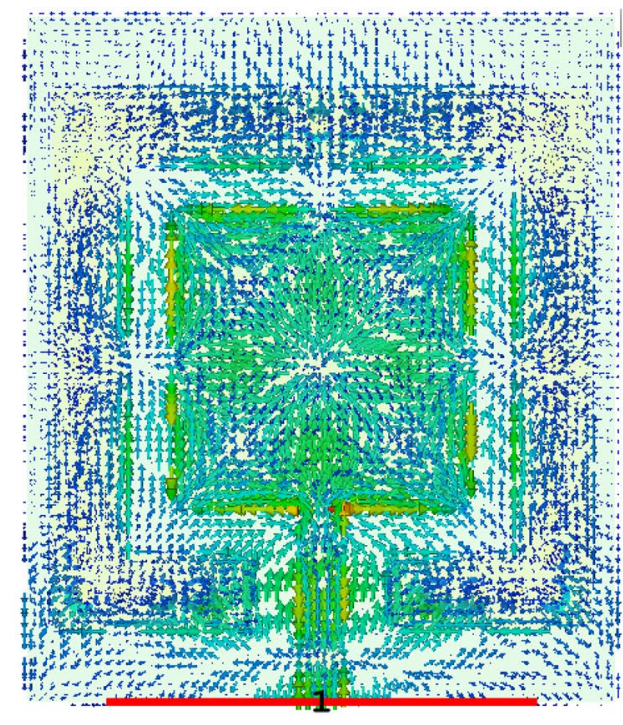

With SIW isolator

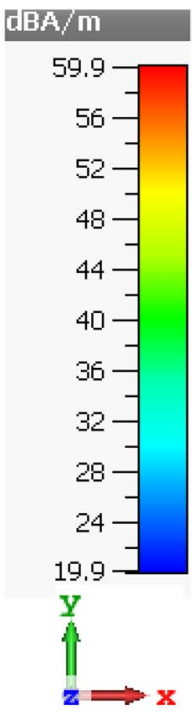

(a) first reflection-coefficient dip of the fundamental $\mathrm{TM}_{01}$ mode at $0.1942 \mathrm{THz}$

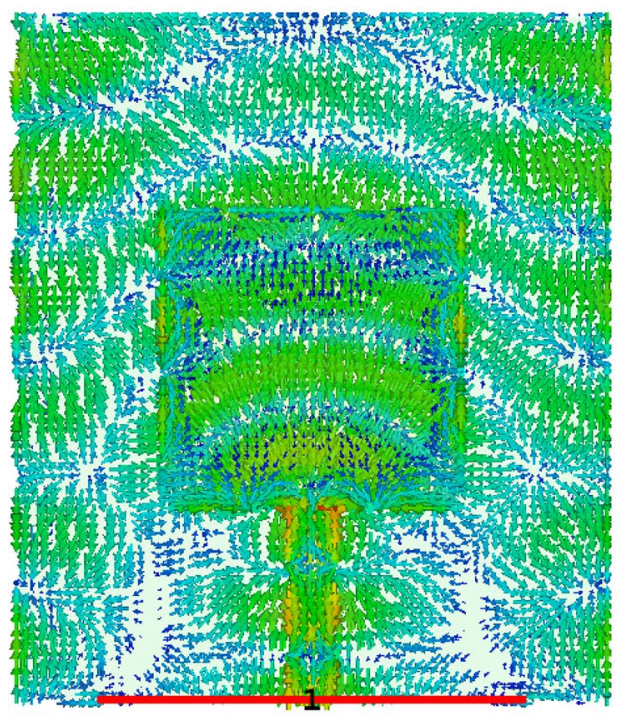

With no SIW isolator
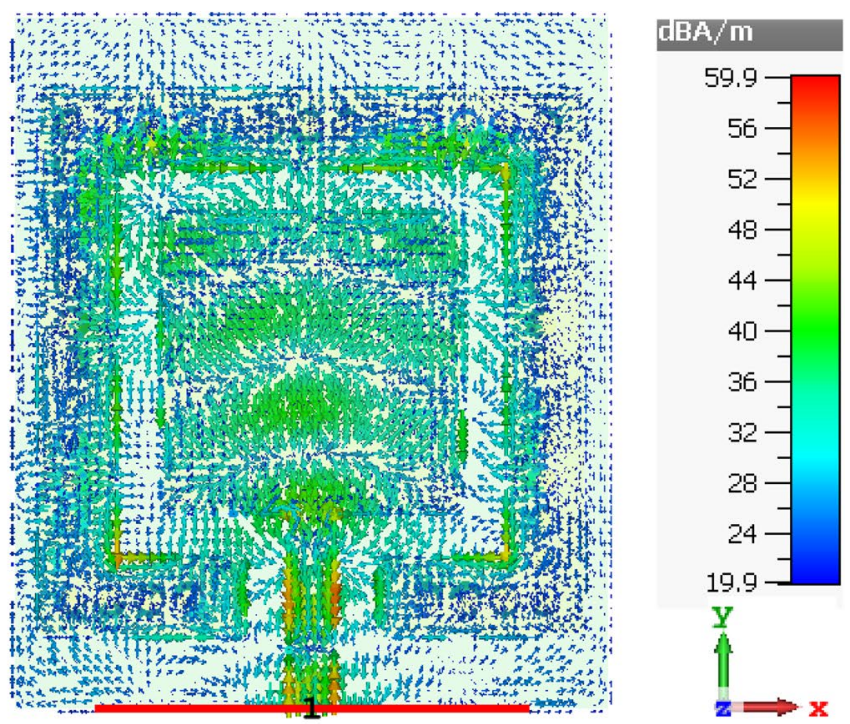

(b) second reflection-coefficient dip of the $\mathrm{TM}_{11}$ mode at $0.1954 \mathrm{THz}$

Figure 4. Surface current density distributions over the reference and SIW antennas at the first and second reflection-coefficient dip frequencies in Fig. 2.

\section{SIW loaded antenna with slots}

Metasurface characteristics are introduced into the patch antenna by the inclusion of periodic array of subwavelength length slots of varying length to realize a wider impedance bandwidth, as shown in Fig. $6^{20}$. These slots exposing the substrate essentially manipulate the electromagnetic (EM) response of the surface. The EM waves impinging on the metasurface induce electric and magnetic dipole moments, which are related to the effective permittivity and permeability of the composite medium. Detailed theoretical analysis of this type of structure is provided $\mathrm{in}^{21}$. To confirm the antenna supports backward waves the CST Microwave Studio, which is a 3D electromagnetic simulation tool, was used to obtain its dispersion characteristics that was computed using ${ }^{22}$ :

$$
\beta p=\cos ^{-1}\left(\frac{1-S_{11} S_{22}+S_{12} S_{21}}{2 S_{21}}\right)
$$

where $\beta$ is the wave propagation constant along the direction of propagation, and $p$ is the length of the unit-cell. Figure 7 shows how the phase through the structure varies with frequency. The dispersion diagram slope is 


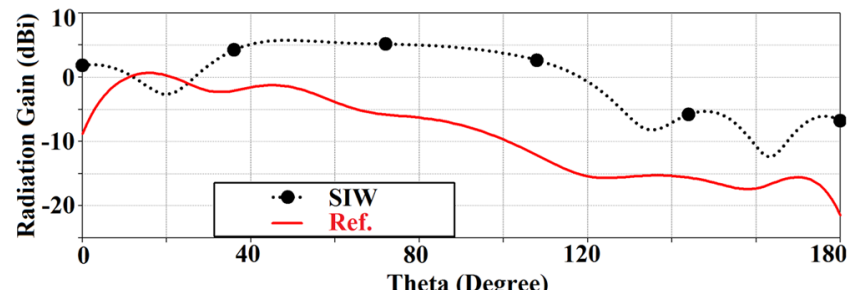

(a) Radiation gain versus azimuth angle at the $1^{\text {st }}$ reflection-coefficient dip at $f_{r_{1}}: 0.1942 \mathrm{THz}$

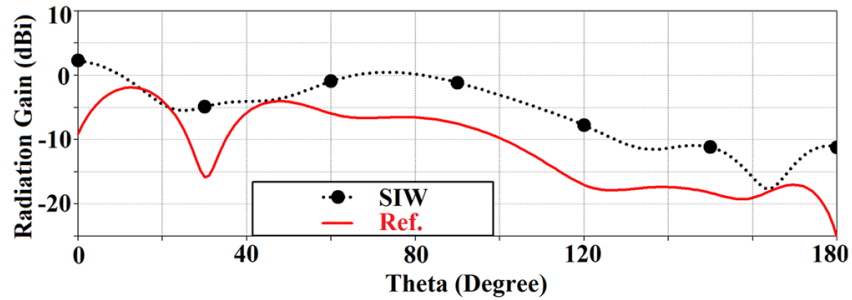

(b) Radiation gain versus azimuth angle at the $2^{\text {nd }}$ reflection-coefficient dip at $f r_{2}: 0.1954 \mathrm{THz}$

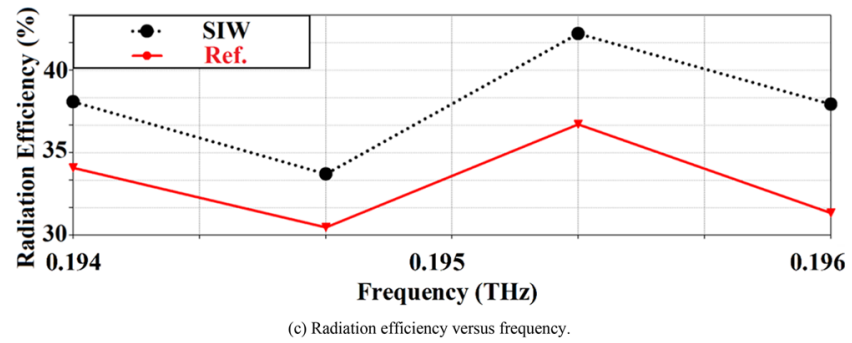

Figure 5. Simulated radiation gain and efficiency plots of the reference and SIW-loaded antennas.

negative between 0.1945 and $0.1951 \mathrm{THz}$. Over this frequency range the signal group and phase velocities are oriented along opposite directions resulting in a backward wave, which is characteristic of metamaterials. This is due to the sub-wavelength slots that control the electromagnetic response of the surface to produce a homogeneous response at 'macroscopic' level. The simplified equivalent circuit of the SIW-loaded slotted antenna in Fig. 8 will be referred to hereon as SIW-loaded MTM. The lumped element values in the equivalent circuit were extracted using the well-established pseudo-inverse technique from S-parameters ${ }^{22,23}$. According to ${ }^{22}$ this structure essentially acts like MTM or composite right/left-handed (CRLH) structure. Magnitude of the equivalent circuit parameters are: $L_{\text {feedline }}=0.021 \mathrm{pH}, L_{\text {patch }}=1.74186 \mathrm{pH}, C_{\text {patch }}=0.408236 \mathrm{pF}, R_{\text {SIW }}=35 \Omega, L_{\text {SIW }}=1 \mathrm{pH}$, $L_{S I W \text {-via }}=2.44918 \mathrm{pH}, \& C_{S I W-\text { fringe }}=0.204575 \mathrm{pF}$.

The array of periodic slots etched on the patch generate multiple resonances whose frequencies can be determined from the dispersion curve when the following condition is satisfied ${ }^{20}$ :

$$
\beta p=\frac{n \pi}{M}\left\{\begin{array}{l}
n=0, \pm 1, \ldots, \pm(M-1), \\
\text { for T - type unit - cell } \\
n=0, \pm 1, \ldots, \pm(M) \\
\text { for } \pi \text { - type unit - cell }
\end{array}\right.
$$

It can be shown from Eq. (2), there are three resonance frequencies which can be excited including the \pm 1 st-order resonances and the zeroth-order resonance at $f_{s e}$. These resonant frequencies are verified from the reflection-coefficient response of the antenna in Fig. 9, which are at $f_{r-1}: 0.1941 \mathrm{THz}, f_{r 0}: 0.1950 \mathrm{THz}$, and $f_{r+1}$ : $0.1957 \mathrm{THz}$. The reflection-coefficient shows significant improvement in impedance match between 0.194 and $0.196 \mathrm{THz}$ with the inclusion of the slots.

The current density distribution in $x y$-plane at the three resonant frequencies is shown in Fig. 10. The current distribution at $f_{r-1}: 0.1941 \mathrm{THz}$ and $f_{r+1}: 0.1957 \mathrm{THz}$ are very similar; however, it is significantly muted at $f_{r 0}$ : $0.1950 \mathrm{THz}$. The size of the antenna is $3.89 \lambda_{0} \times 4.54 \lambda_{0} \times 0.08 \lambda_{0}$ when operated at $f_{r-1}: 0.1941 \mathrm{THz}$.

Comparison of the radiation plots of the reference, SIW-loaded, and SIW-loaded slotted antennas are shown in Fig. 11. It is evident that the SIW-loaded MTM patch antenna exhibits significantly improved performance over the other two antennas. The optimum radiation gain of the SIW-loaded MTM antenna at resonance modes - 1st $\left(f_{r-1}: 0.1941 \mathrm{THz}\right)$, zeroth $\left(f_{r 0}: 0.1950 \mathrm{THz}\right)$, and $+1 \mathrm{st}\left(f_{r+1}: 0.1957 \mathrm{THz}\right)$ are $8.05 \mathrm{dBi} @ 87.7^{\circ}, 8.0 \mathrm{dBi} @ 51.6^{\circ}$, and $7.92 \mathrm{dBi} @ 59.3^{\circ}$, respectively. Besides the gain, the radiation efficiency at resonance modes - 1st, zeroth, and +1 st are $45 \%, 45 \%$, and $47 \%$, respectively. In the case of $f_{r-1}: 0.1941 \mathrm{THz}$ and $f_{r+1}: 0.1957 \mathrm{THz}$, the gain of the reference antenna is comparable to the other two antennas, but this is over a very narrow azimuth range between $12^{\circ}$ and $26^{\circ}$. Total dimensions of the three antennas are identical. 


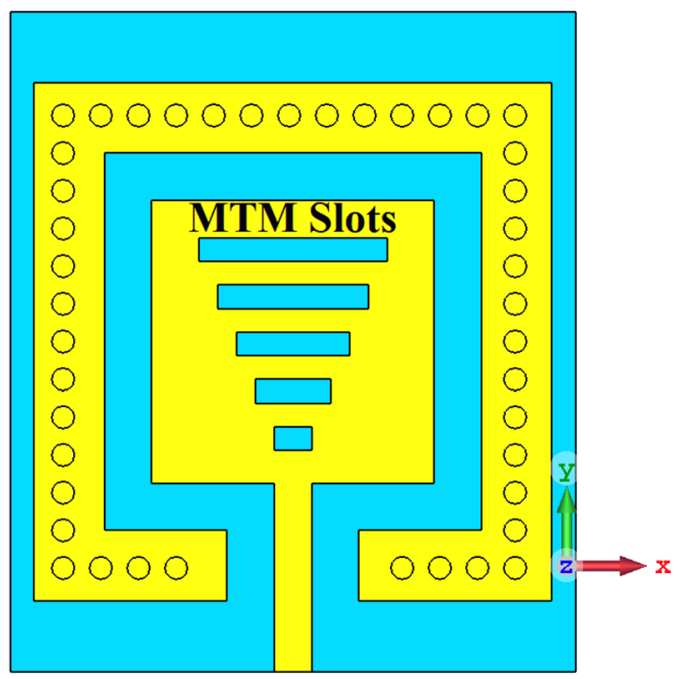

(a)

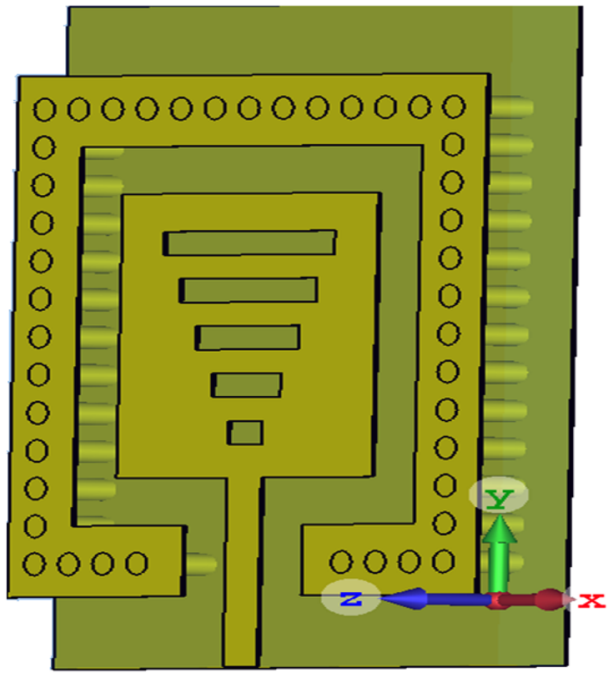

(b)

Figure 6. (a) front view, (b) schematic view of the SIW-loaded slotted antenna.

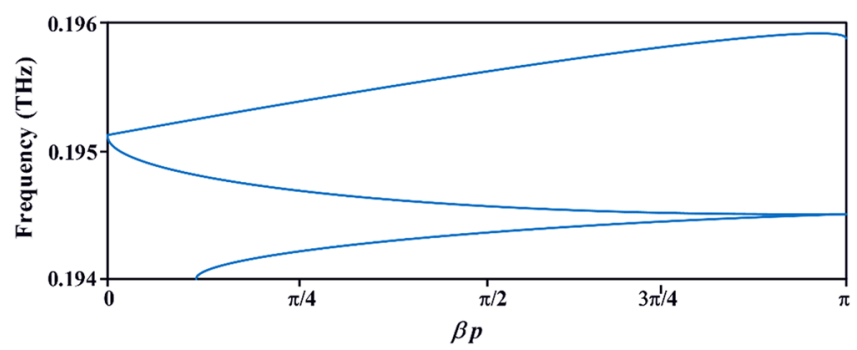

Figure 7. Dispersion diagram of the SIW-loaded slotted antenna.

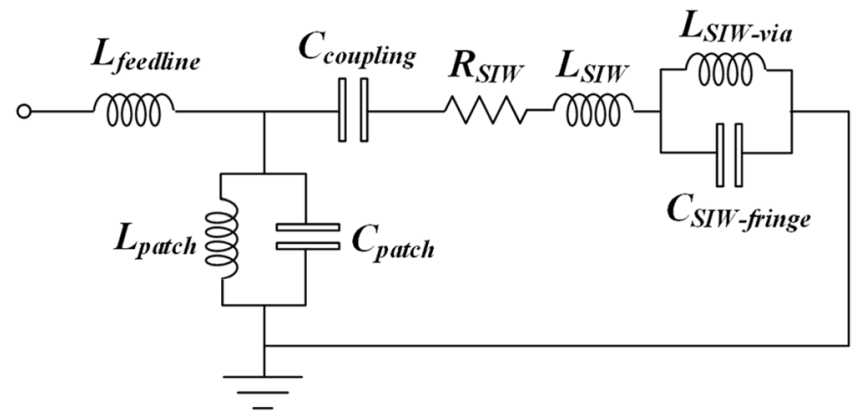

Figure 8. The simplified equivalent circuit of the SIW-loaded slotted antenna.

\section{Array configuration of the proposed SIW-loaded MTM antenna for sub-THz applications}

In this section $2 \times 3$ antenna arrays are realized based on the standard reference, SIW-loaded, and SIW-loaded MTM patch antenna. The main issue encountered in an array structure is the adverse effect of mutual coupling between the radiating elements constituting the array. Several techniques previously studied and proposed are exemplified in ${ }^{23-28}$ but the size of the array matrix investigated in all cases reported was limited to $1 \times 2$. The techniques proposed previously include the use of air gap between radiator and ground-plane or the implementation of ground-plane defection. In all cases reported the isolation achieved is limited and is restricted over narrow frequency band. 


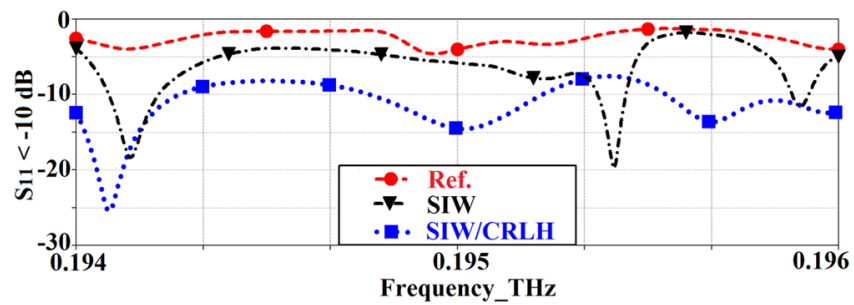

Figure 9. Simulated reflection-coefficient response of the reference, SIW-loaded, and SIW-loaded MTM (CRLH) antennas.

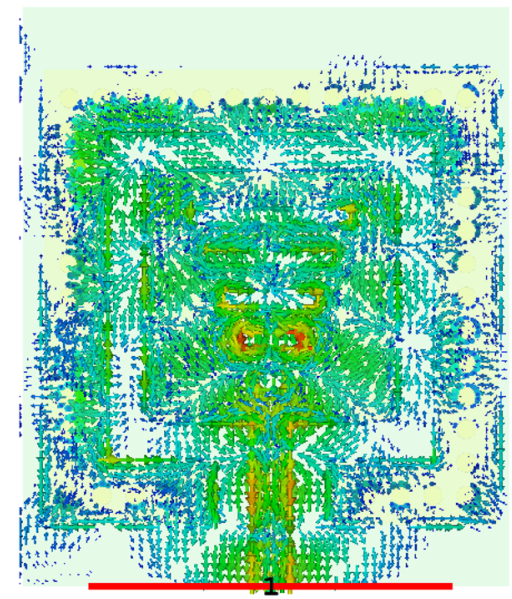

(a) $0.1941 \mathrm{THz}$

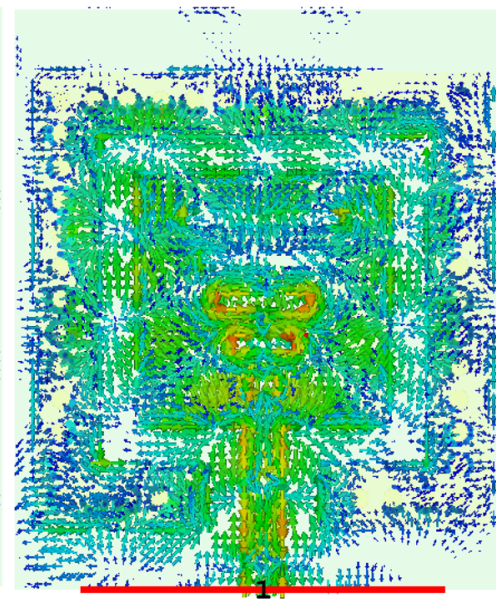

@ $0.1950 \mathrm{THz}$
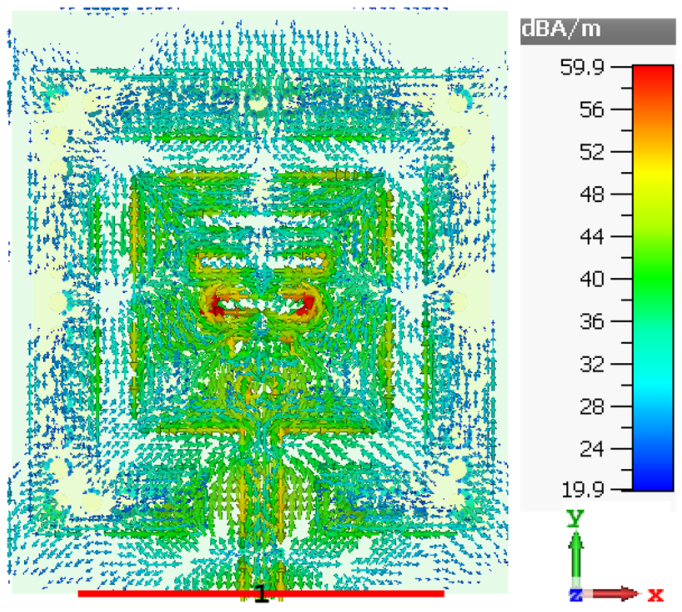

(a) $0.1957 \mathrm{THz}$

Figure 10. Surface current density distributions over the SIW-loaded MTM antenna at the first, second and third resonance frequencies.

In small-sized antenna array structures it is very difficult to supress the mutual coupling effects resulting from surface wave interaction between the radiation elements due to their proximity. Proposed here is a simple but effective mutual coupling reduction technique based on SIW isolator as described in "SIW loaded antenna with slots" section. The prototype of the SIW-loaded MTM antenna is shown in Fig. 12, where the SIW isolator is wrapped around each patch to significantly reduce propagation of surface waves and minimise substrate loss. The prototype antenna array was coated with $500 \mathrm{~nm}$ thick film of Graphene. The identical patches in the array have dimensions of $3 \times 3 \times 0.125 \mathrm{~mm}^{3}$. The dimension of the $2 \times 3$ antenna array is $20 \times 13.5 \times 0.125 \mathrm{~mm}^{3}$. Dimensions of the optimized structural parameters are tabulated in Table 2.

\section{Results and discussions}

The simulated and measured impedance bandwidth of the standard reference, SIW-loaded, and SIW-loaded MTM $2 \times 3$ antenna arrays are shown in Fig. 13. CST Microwave Studio was used obtain the simulation results. Keysight PNA Network Analyzer with a frequency extender was used to measure the reflection-coefficient of the antenna. These results clearly show that the SIW isolator has a significant impact on the impedance matching and bandwidth of the array over the standard reference antenna array. Further improvement is achieved by incorporating sub-wavelength slots in the patch antennas. The experimental results show that with SIW-loading the impedance matching is improved by $10 \mathrm{~dB}$ on average from 190 to $200 \mathrm{GHz}$ over the reference antenna array. By incorporating both SIW-loading and sub-wavelength slots the impedance matching also improves on average by $14 \mathrm{~dB}$ from 190 to $200 \mathrm{GHz}$ compared to the reference array antenna. There is generally good agreement between the simulated and measured results. The discrepancy in the results is attributed to manufacturing tolerance and unaccounted loss in the simulation model.

The transmission-coefficient response of the standard reference, SIW-loaded, and SIW-loaded MTM $2 \times 3$ antenna array are shown in Fig. 14. These results demonstrate the effectiveness of the proposed technique of using SIW isolator in suppressing the surface-wave propagations and reducing substrate loss to mitigate mutual coupling effects without increasing the overall size of the array structure. Experimental results show that compared to the reference antenna array the SIW-loaded array is shown to improve the isolation on average by $17 \mathrm{~dB}$ from 190 to $200 \mathrm{GHz}$. Also, by incorporating sub-wavelength slots the SIW-loaded MTM array is shown to suppress mutual coupling on average by more than $28 \mathrm{~dB}$ across $190 \mathrm{GHz}$ to $200 \mathrm{GHz}$ compared to the reference array antenna.

The simulated and measured radiation gain and efficiency of the standard reference, SIW-loaded, and SIWloaded MTM (CRLH) array are shown in Fig. 15. Experimental results show that compared to the reference array 


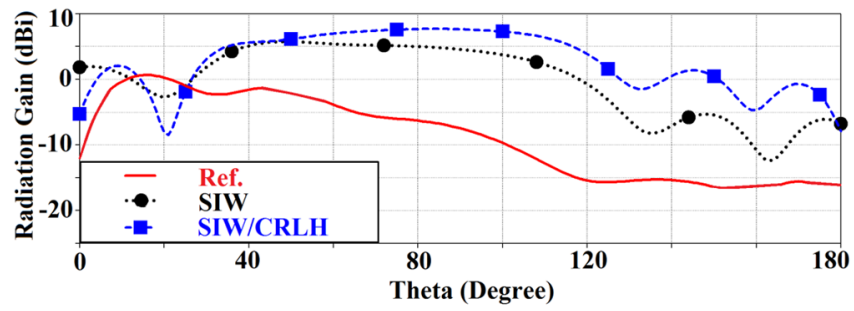

(a) Radiation gain versus azimuth angle at the $1^{\text {st }}$ reflection-coefficient dip of $f_{r-1}: 0.1941 \mathrm{THz}$

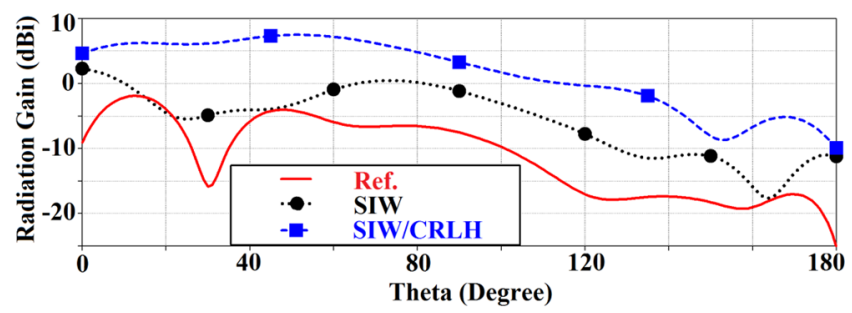

(b) Radiation gain versus azimuth angle at the $2^{\text {nd }}$ reflection-coefficient dip of $f_{r 0}: 0.1950 \mathrm{THz}$

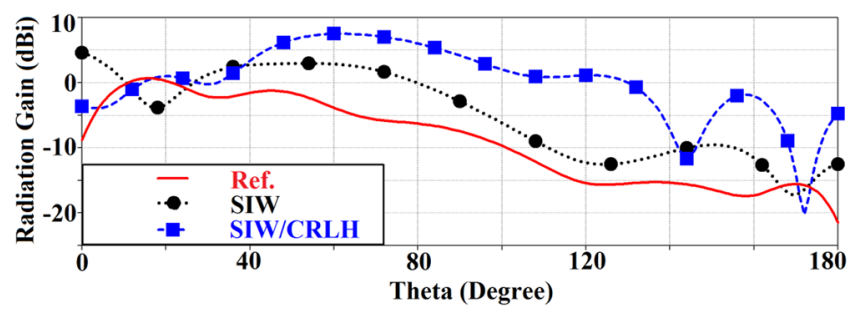

(c) Radiation gain versus azimuth angle at the $3^{\text {rd }}$ reflection-coefficient dip of $f_{r+1}: 0.1957 \mathrm{THz}$

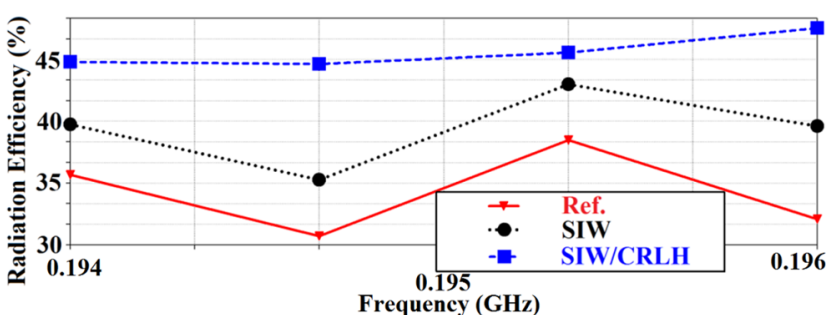

(d) Radiation efficiency versus frequency.

Figure 11. Simulated radiation gain and efficiency of the reference, SIW-loaded, and SIW-loaded MTM (CRLH) antennas.

\begin{tabular}{|l|l|}
\hline Parameters & Dimension $(\mathbf{m m})$ \\
\hline Patch length & 3 \\
\hline Patch width & 3 \\
\hline Feed line length & 2 \\
\hline Feed line width & 0.4 \\
\hline SIW isolator width & 0.75 \\
\hline Number of tapered rectangular slots & 5 \\
\hline Slot lengths (sub-wavelength) & $0.48,0.56,0.84,1.12,1.40$ \\
\hline Slot width & 0.25 \\
\hline Gap between patch and isolator & 0.5 \\
\hline Gap between feed line and isolator & 0.5 \\
\hline Diameter of vias & 0.25 \\
\hline Gap between vias & 0.20 \\
\hline Total length & 20 \\
\hline Total width & 13.5 \\
\hline Thickness of graphene layer & 0.0005 \\
\hline Substrate thickness & 0.125 \\
\hline Edge-to-edge gap between adjacent patches & 3.5 \\
\hline Edge-to-edge gap between SIW isolators & 1 \\
\hline
\end{tabular}

Table 2. Structural dimensions of the antenna. 


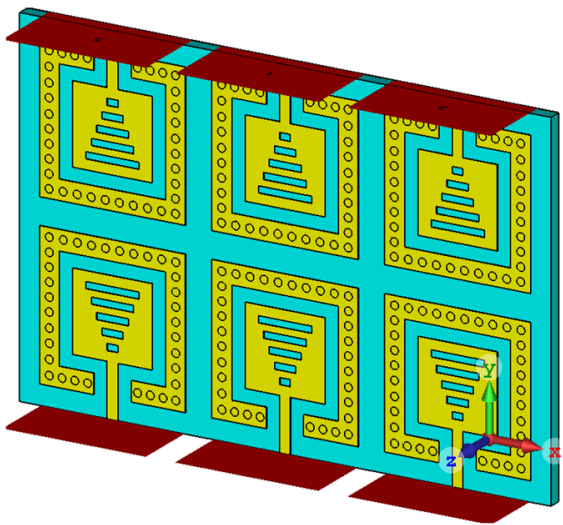

(a)

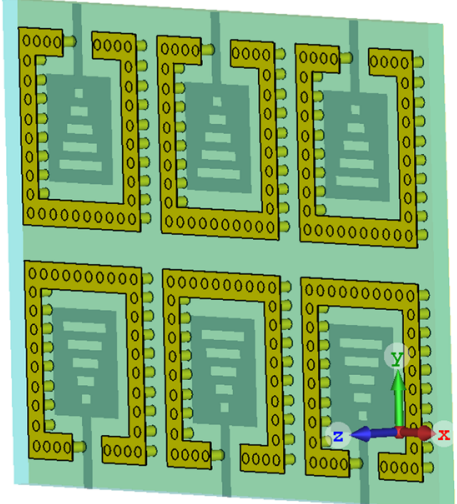

(c)

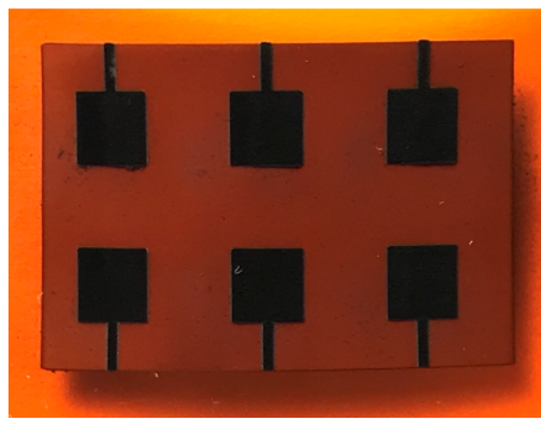

(e)
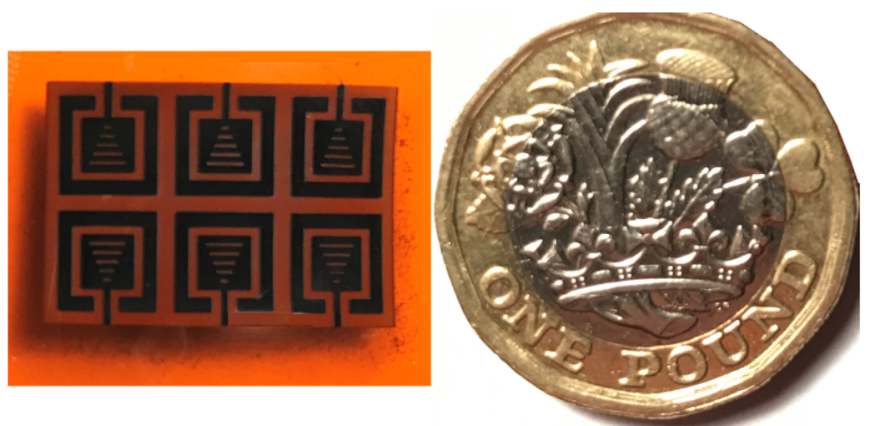

(g)

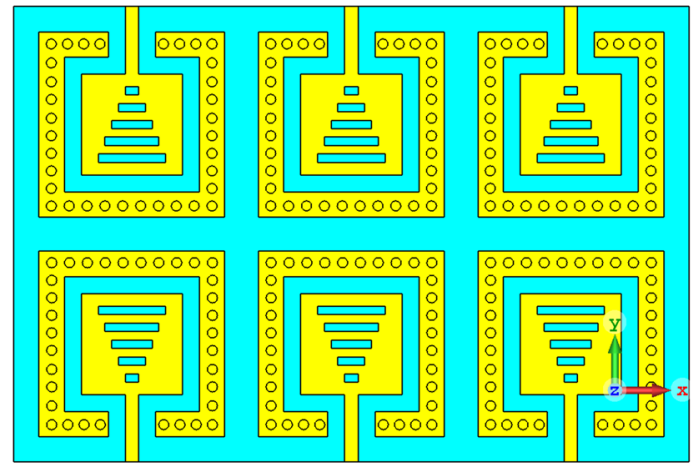

(b)

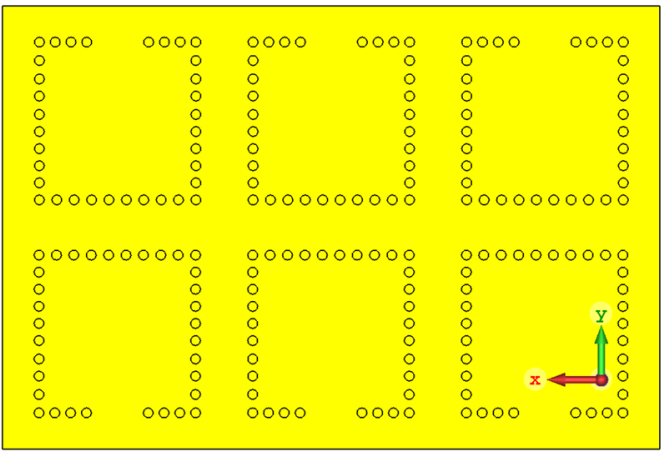

(d)

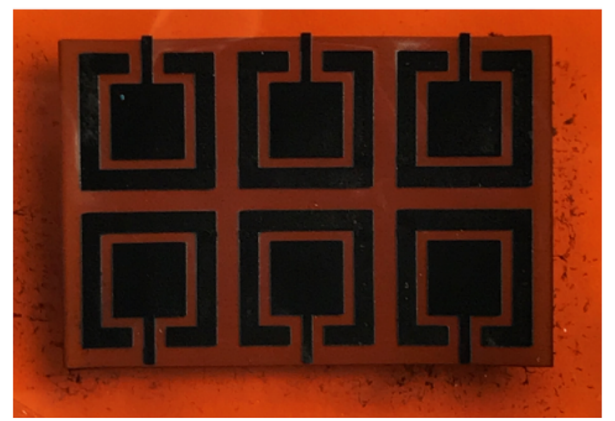

(f)

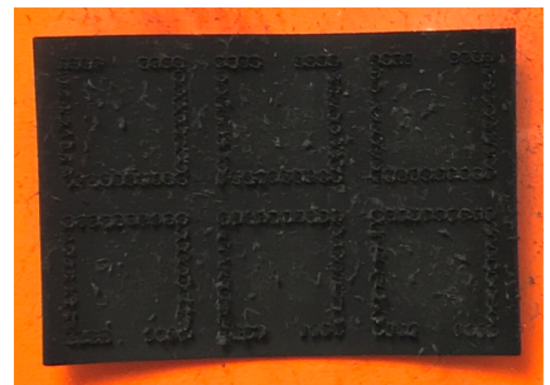

(h)

Figure 12. Proposed Graphene coated $2 \times 3$ antenna array based on SIW-loaded MTM patches, (a) isometric view, (b) front view, (c) SIW isolators framing the radiation patches, (d) back view (ground-plane), and (e-h) the fabricated antenna array prototype of the reference, SIW-loaded, SIW-loaded MTM antenna, and the ground-plane, respectively. 


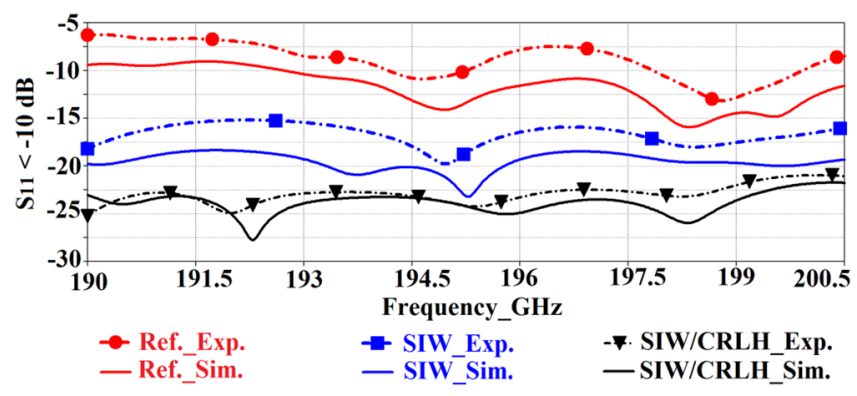

Figure 13. Simulated and experimental results of the reflection-coefficient of the reference, SIW-loaded, and SIW-loaded MTM (CRLH) antenna arrays.

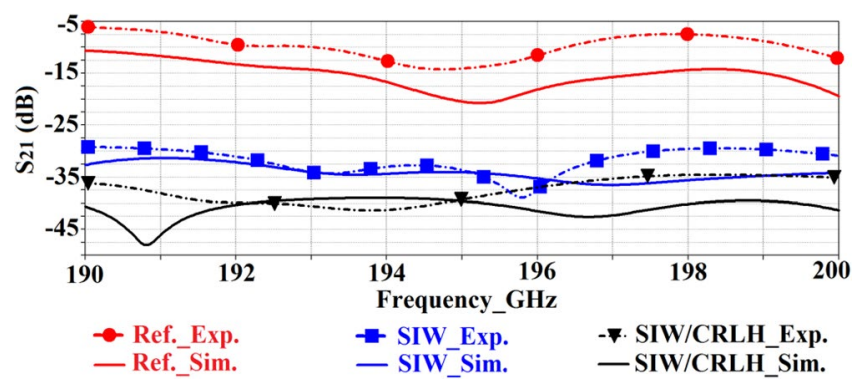

Figure 14. Simulated and experimental results of the transmission-coefficient $\left(\mathrm{S}_{21}\right)$ of the reference, SIWloaded, and SIW-loaded MTM (CRLH) antenna arrays.

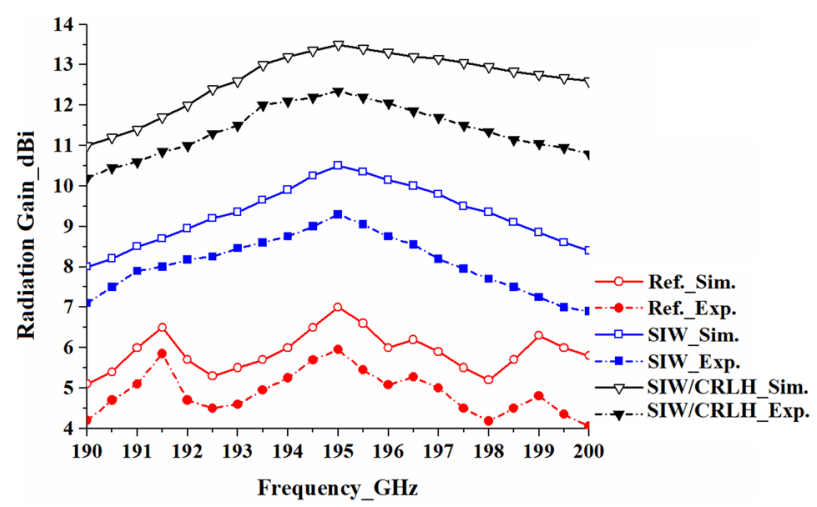

(a) Radiation gain

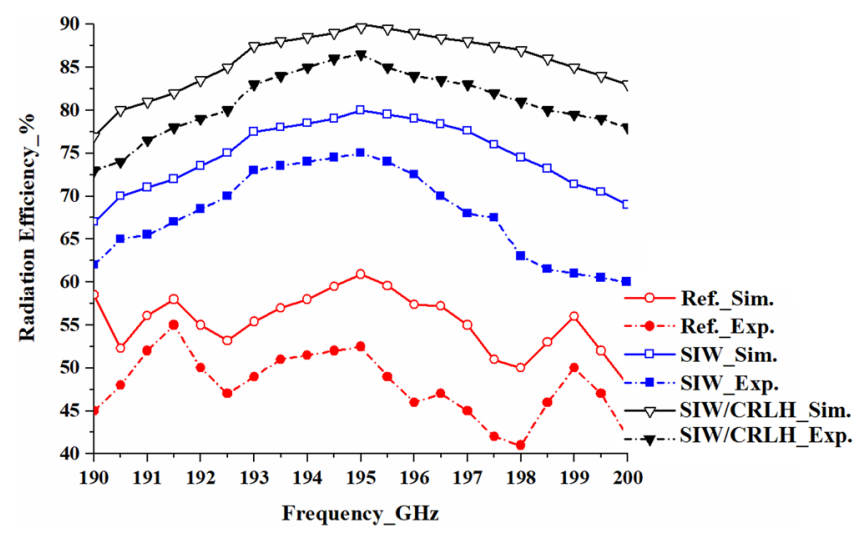

(b) Radiation efficiency

Figure 15. Simulated and experimental results of the radiation gain and efficiency as a function of frequency of the reference, SIW-loaded, and SIW-loaded MTM (CRLH) antenna arrays. 


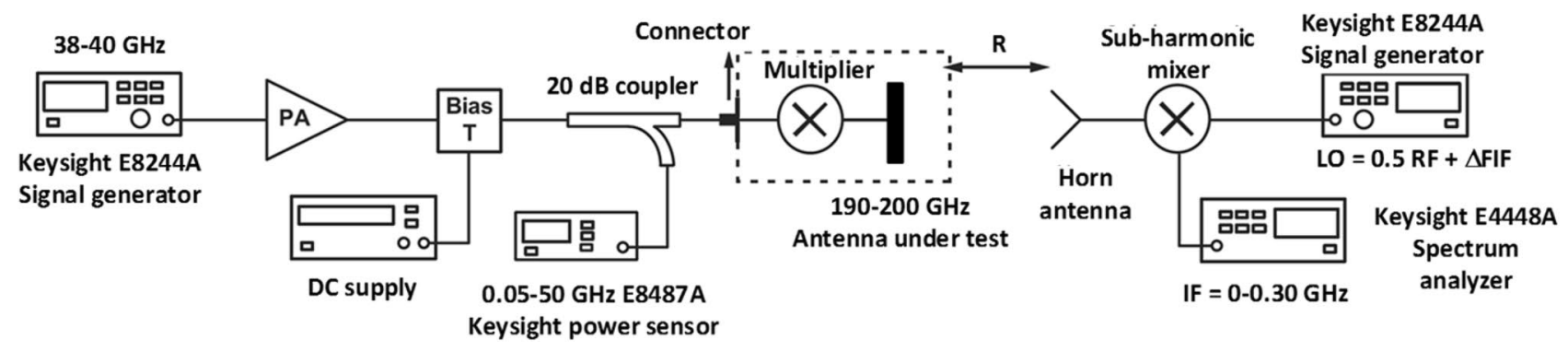

Figure 16. Set-up to measure the array's radiation patterns.

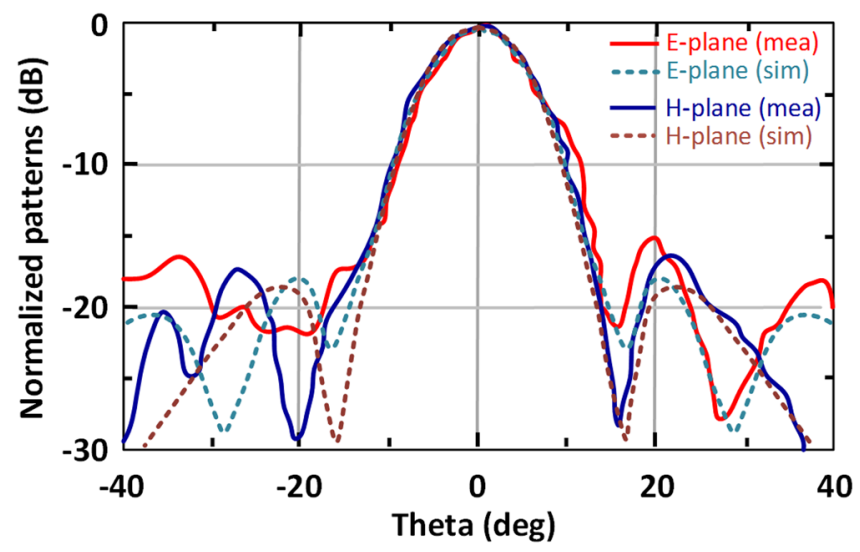

(a)

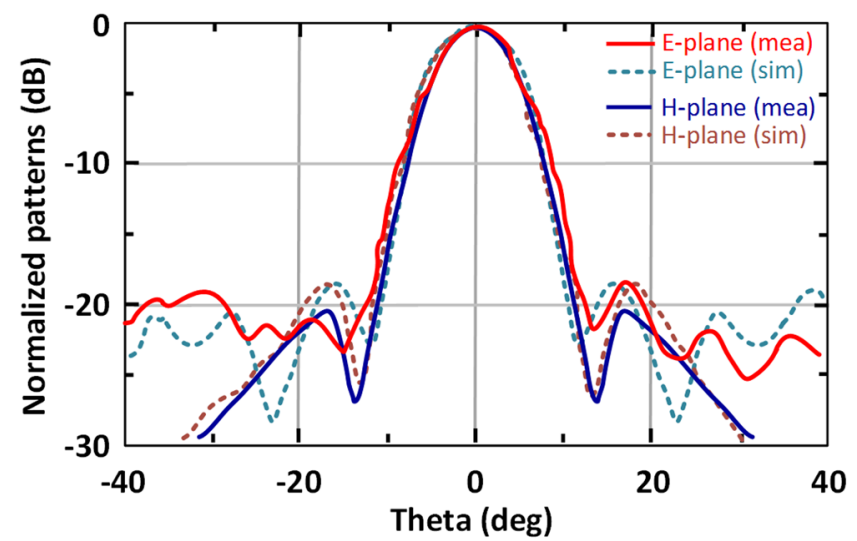

(b)

Figure 17. Measured and simulated radiation pattern of the $2 \times 3$ SIW-loaded MTM antenna arrays in E-plane and H-plane at (a) $190 \mathrm{GHz}$, and (b) $200 \mathrm{GHz}$.

the SIW-loaded array's gain and efficiency improve on average by $2.8 \mathrm{dBi}$ and 23\%, respectively, between 190 and $200 \mathrm{GHz}$. With SIW-loaded MTM the average improvement in gain and efficiency over the reference array is 6.3 $\mathrm{dBi}$ and 34\%, respectively, between 190 and $200 \mathrm{GHz}$. These results demonstrate the array's effective aperture is enlarged by incorporating sub-wavelength slots, which has the benefit of not affecting the antenna's dimensions.

The set-up to measure the far-field radiation patterns of the SIW-loaded MTM antenna arrays is shown in Fig. 16. In transmit mode we used a 2 -way power splitter in conjunction with two 3 -way power splitters. It was necessary to ensure the lengths were such that the phase at the antenna terminals were identical. This arrangement was used to split equally the input sub-THz signal applied to the $2 \times 3$ array. Conversely, in the receive mode we used two 3 -way power combiners in combination with a 2 -way power combiner. This arrangement combined the received signal power from the $2 \times 3$ array. The output from the array in receive mode was connected to Keysight E4448A spectrum analyser. Keysight E8244A signal generator was used to produce a signal that was adjustable in the frequency range $38-40 \mathrm{GHz}$ with enough power $(10-20 \mathrm{dBm})$ for the mixer to generate a set of harmonics simultaneously. The 5th harmonic was used as it was in the desired frequency range of 190-200 GHz. 


\begin{tabular}{|c|c|c|c|c|c|}
\hline References & Type & Bandwidth [freq. range] & Gain $(\mathbf{d B i})$ & Eff. (\%) & Size $\left(\mathbf{m m}^{3}\right)$ \\
\hline 29 & Bowtie-slot & $15 \mathrm{GHz}[90-105]$ & $\begin{array}{l}\operatorname{Max} \\
-1.78\end{array}$ & - & $0.71 \times 0.31 \times 0.65$ \\
\hline 30 & Differential-fed & $20 \mathrm{GHz}[50-70]$ & $\begin{array}{c}\text { Max } \\
-3.2\end{array}$ & - & $1.5 \times 1.5 \times 0.3$ \\
\hline 31 & Ring-shaped monopole & $20 \mathrm{GHz}[50-70]$ & Max. 0.02 & Max. 35 & - \\
\hline 32 & Circular open-loop & $10 \mathrm{GHz}[57-67]$ & $\begin{array}{l}\text { Max } \\
-4.4 \\
\end{array}$ & - & $1.8 \times 1.8 \times 0.3$ \\
\hline 33 & AMC embedded squared slot antenna & $51 \mathrm{GHz}[15-66]$ & Max. 2 & - & $1.44 \times 1.1$ \\
\hline 34 & Monopole & $25 \mathrm{GHz}[45-70]$ & Max. 4.96 & - & $1.953 \times 1.93 \times 0.25$ \\
\hline 35 & Loop antenna & $4 \mathrm{GHz}[65-69]$ & Max. 8 & Max. 96.7 & $0.7 \times 1.25$ \\
\hline 36 & Dipole-antenna & $7 \mathrm{GHz}[95-102]$ & Max. 4.8 & - & - \\
\hline 37 & Tab monopole & $30 \mathrm{GHz}[45-75]$ & Max. 0.1 & Max. 42 & $1.5 \times 1$ \\
\hline 38 & Patch fed higher order mode DRA & $25 \mathrm{GHz}[330-355]$ & Max. 7.9 & Max. 74 & $0.2 \times 0.5$ \\
\hline 39 & On-chip 3D (Yagi like concept) & $40 \mathrm{GHz}[320-360]$ & Max. 10 & Max. 80 & $0.7 \times 0.7 \times 0.43$ \\
\hline 40 & Half-mode cavity fed DRA & $15 \mathrm{GHz}[125-140]$ & Max. 7.5 & Max. 46 & $0.8 \times 0.9 \times 1.3$ \\
\hline 41 & Slot fed stacked DRA & $10 \mathrm{GHz}[125-135]$ & Max. 4.7 & Max. 43 & $0.9 \times 0.8 \times 1.5$ \\
\hline 42 & DRA & $20 \mathrm{GHz}[120-140]$ & Max. 2.7 & Max. 43 & $0.9 \times 0.8 \times 0.6$ \\
\hline 43 & $8 \times 8$ Magneto-electric dipole antenna array & $14.7 \mathrm{GHz}[130.3-145]$ & Max. 20.5 & Max. 59.2 & $32 \times 20 \times 0.818$ \\
\hline 44 & $4 \times 1$ Patch antenna array & $32 \mathrm{GHz}[259-291]$ & Max. 5.2 & - & $2.47 \times 1.53 \times 0.675$ \\
\hline 45 & $\begin{array}{l}2 \times 1 \text { Octagonal shorted annular ring antenna } \\
\text { array }\end{array}$ & $17 \mathrm{GHz}[303-320]$ & Max. 4.1 & Max. 38 & $0.55 \times 0.5 \times 0.3$ \\
\hline This work & SIW-loaded MTM & $10 \mathrm{GHz}[190-200]$ & Max. 12.2 & Max. 86 & $20 \times 13.5 \times 0.125$ \\
\hline
\end{tabular}

Table 3. Salient features of the proposed SIW-loaded MTM antenna array compared with recent publications.

At the receiver, the received signal is mixed with signal from Keysight E8244A with a frequency which was offset from the transmitter local oscillator, and this offset frequency $\left(\Delta \mathrm{F}_{\mathrm{IF}}\right)$ determines the system harmonic number, which is used $\left(5 \Delta \mathrm{F}_{\mathrm{IF}}\right)$. The simulated and measured patterns of the $2 \times 3$ SIW-loaded MTM antenna arrays in the E- and $\mathrm{H}$-planes at $190 \mathrm{GHz}$ and $200 \mathrm{GHz}$ are shown in Fig. 17. CST Microwave Studio was used obtain the simulation results. The measured results agree well with the simulations. The radiation pattern is stable and symmetrical within the operating band of the array. Due to the unpredictable reflection from the fixtures and measurement setup near the antenna under test, a series of small ripples can be seen in the measured results.

Performance parameters of the proposed SIW-loaded MTM antenna array is compared with other recently published millimeter-wave and sub-THz antennas in Table 3. The comparison shows that the proposed antenna array has comparable gain and radiation efficiency to the cited references, which confirm the effectiveness of the proposed approach. It is less complex and cost effective to implement and realize in practice for mass production, which makes it a viable candidate for applications to sub-terahertz wireless systems.

\section{Conclusion}

We have demonstrated the use of a Graphene-based antenna at sub-THz and the effectiveness of the proposed technique of framing each radiator with SIW. This technique is shown here to reduce surface wave propagations and suppressing near-field mutual coupling between the radiators in an array. The consequence of this is significant improvement in the array's performance in terms of impedance matching and radiation performance which is achieved without compromising the footprint of the antenna array. Further improvement in performance was demonstrated by incorporating sub-wavelength slots in the patch radiators. The feed mechanism employed is however considered to be unfeasible for a large array, where aperture coupling would be more appropriate.

Received: 30 November 2020; Accepted: 1 April 2021

Published online: 13 May 2021

\section{References}

1. Nagatsuma, T., Ducournau, G. \& Renaud, C. C. Advances in terahertz communications accelerated by photonics. Nat. Photonics 10, 371-379 (2016).

2. Akyildiz, I. F., Jornet, J. M. \& Han, C. Terahertz band: Next frontier for wireless communications. Physical Communication 12, 16-32 (2014).

3. Song, H. J. \& Nagatsuma, T. Present and future of terahertz communications. IEEE Trans. Terahertz Sci. Technol. 1, 256-263 (2011).

4. Al Hadi, R. et al. A 1 k-pixel video camera for 0.7-1.1 terahertz imaging applications in 65-nm CMOS. IEEE J. Solid-State Circuits 47, 2999-3012 (2012).

5. Walther, M. et al. Terahertz conductivity of thin gold films at the metal-insulator percolation transition. Phys. Rev. B 76, 125408 (2007).

6. Hanson, G. Radiation efficiency of nano-radius dipole antennas in the microwave and far-infrared regimes. IEEE Antennas Propag. Mag. 50, 66-77 (2008).

7. Laman, N. \& Grischkowsky, D. Terahertz conductivity of thin metal films. Appl. Phys. Lett. 93, 051105 (2008).

8. Kuan, T., Inoki, C., Oehrlein, G., Rose, K., Zhao, Y. P., Wang, G. C. et al. Fabrication and performance limits of sub-0.1 $\mu$ m Cu interconnects. In MRS Proceedings (2000). 
9. Lee, D.-K. et al. Highly sensitive and selective sugar detection by terahertz nano-antennas. Sci. Rep. 5, 15459 (2015).

10. Razzari, L. et al. Terahertz dipole nanoantenna arrays: resonance characteristics. Plasmonics 8, 133-138 (2013).

11. Feuillet-Palma, C., Todorov, Y., Vasanelli, A. \& Sirtori, C. Strong near field enhancement in THz nano-antenna arrays. Sci. Rep. 3, 1361 (2013).

12. Lier, E., Werner, D. H., Scarborough, C. P., Wu, Q. \& Bossard, J. A. An octave-bandwidth negligible-loss radio frequency metamaterial. Nat. Mater. 10, 216-222 (2011).

13. Pfeiffer, U. R. et al. Current status of terahertz integrated circuits: from components to systems. In IEEE BiCMOS and Compound Semiconductor Integrated Circuits and Technology Symposium (BCICTS), 107 (2018).

14. Dan, I. et al. A Terahertz wireless communication link using a superheterodyne approach. IEEE Trans. Terahertz Sci. Technol. 10(1), 1-12 (2020).

15. Pendry, J. B., Martin-Moreno, L. \& GarciaVidal, F. J. Mimicking surface plasmons with structured surfaces. Science 305, 847-848 (2004).

16. Shimada, Y., Iida, H. \& Kinoshita, M. Recent research trends of terahertz measurement standards. IEEE Trans. Terahertz Sci. Technol. 5(6), 1166-1172 (2015).

17. Bozzi, M., Pierantoni, L. \& Bellucci, S. Applications of graphene at microwave frequencies. Radioengineering 24(3), 661-669 (2015).

18. Yin, Y., Yao, Y., Liu, X., Qi, L., Chen, Z., Yu, J., \& Chen, X. Research on terahertz reflectarray based on graphene surface and PET substrate. In 10th UK-Europe-China Workshop on Millimetre Waves and Terahertz Technologies (UCMMT), 1-3 (2017).

19. Zhao et al., "Replacing the metal electrodes in triboelectric nanogenerators: High-performance laser-induced graphene electrodes", Nano Energy, 75, 104958 (2020).

20. Finch, M. F. \& Lail, B. A. Multi-coupled resonant splitting with a nano-slot metasurface and PMMA phonons. In Proc. SPIE 9547, Plasmonics: Metallic Nanostructures and Their Optical Properties XIII, 954710, 28, 1-6 (2015).

21. Martini, E., Mencagli, M. Jr. \& Maci, S. Metasurface transformation for surface wave control. Philos. Trans. R. Soc. A 373, 20140355 (2015).

22. Caloz, C. \& Itoh, T. Electromagnetic Metamaterials: Transmission Line Theory and Microwave Applications (Wiley, 2005).

23. Kou, N., Shi, Y. \& Li, L. New equivalent circuit analysis and synthesis for broadband composite right/left-handed transmission line metamaterials. ACES J. 31(8), 884-893 (2016).

24. Farsi, S., Schreurs, D. \& Nauwelaers, B. Mutual coupling reduction of planar antenna by using a simple microstrip U-section. IEEE Ant. Wirel. Propag. Lett. 11, 1501-1503 (2012).

25. Alsath, M. G., Kanagasabai, M. \& Balasubramanian, B. Implementation of slotted meander line resonators for isolation enhancement in microstrip patch antenna arrays. IEEE Antennas Wirel. Propag. Lett. 12, 15-18 (2013).

26. Shafique, M. F., Qamar, Z., Riaz, L., Saleem, R. \& Khan, S. A. Coupling suppression in densely packed microstrip arrays using metamaterial structure. Microw. Opt. Technol. Lett. 57(3), 759-763 (2015).

27. Ghosh, J., Ghosal, S., Mitra, D. \& Chaudhuri, S. R. B. Mutual coupling reduction between closely placed microstrip patch antenna using meander line resonator. Progress in Electromag. Research Lett. 59, 115-122 (2016).

28. Yang, Xu., Liu, Y., Yun-Xue, Xu. \& Gong, S.-X. Isolation enhancement in patch antenna array with fractal UC-EBG structure and cross slot. IEEE Antennas Wirel. Propag. Lett. 16, 2175-2178 (2017).

29. Khan, M. S., Tahir, F. A. \& Cheema, H. M. Design of bowtie-slot on-chip antenna backed with E-shaped FSS at 94 GHz. In $10 t h$ European Conference on Antennas and Propagation (EuCAP), Davos, 1-3 (2016).

30. Wang, L. \& Sun, W. Z. A 60-GHz differential-fed circularly polarized on-chip antenna based on $0.18-\mu \mathrm{m}$ COMS technology with AMC structure. In IET International Radar Conference, Hangzhou, 1-4 (2015).

31. Huang, H. T., Yuan, B., Zhang, X. H., Hu, Z. F. \& Luo, G. Q. A circular ring-shape monopole on-chip antenna with artificial magnetic conductor. In Asia-Pacific Microwave Conference (APMC), Nanjing, 1-3 (2015).

32. Bao, X., Guo, Y. \& Xiong, Y. 60-GHz AMC-based circularly polarized on-chip antenna using standard 0.18- $\mu \mathrm{m}$ CMOS Technology. IEEE Trans. Antennas Propag. 60(5), 2234-2241 (2012).

33. Lin, F. \& Ooi, B. L. Integrated millimeter-wave on-chip antenna design employing artificial magnetic conductor. In IEEE International Symposium on Radio-Frequency Integration Technology (RFIT), Singapore, 174-177 (2009).

34. Upadhyay, S. \& Srivastava, S. A $60-\mathrm{GHz}$ on-chip monopole antenna using silicon technology. In IEEE Applied Electromagnetics Conference (AEMC), Bhubaneswar, 1-2 (2013).

35. Song, Y., Wu, Y., Yang, J. \& Kang, K. The design of a high gain on-chip antenna for SoC application. In IEEE MTT-S International Microwave Workshop Series on Advanced Materials and Processes for RF and THz Applications (IMWS-AMP), Suzhou, 1-3 (2015).

36. Nafe, M., Syed, A. \& Shamim, A. Gain enhancement of low profile on-chip dipole antenna via artificial magnetic conductor at 94 GHz. In 9th European Conference on Antennas and Propagation (EuCAP), Lisbon, 1-3 (2015).

37. Yang, W., Ma, K., Yeo, K. S. \& Lim, W. M. A 60 GHz on-chip antenna in standard CMOS silicon technology. In IEEE Asia Pacific Conference on Circuits and Systems, Kaohsiung, 252-255 (2012).

38. Li, C. \& Chiu, T. 340-GHz low-cost and high-gain on-chip higher order mode dielectric resonator antenna for THz applications. IEEE Trans. Terahertz Sci. Technol. 7(3), 284-294 (2017).

39. Deng, X., Li, Y., Liu, C., Wu, W. \& Xiong, Y. $340 \mathrm{GHz}$ on-chip 3-D antenna with $10 \mathrm{dBi}$ gain and $80 \%$ radiation efficiency. IEEE Trans. Terahertz Sci. Technol. 5(4), 619-627 (2015).

40. Hou, D. et al. D-band on-chip higher-order-mode dielectric-resonator antennas fed by half-mode cavity in CMOS technology. IEEE Antennas Propag. Mag. 56(3), 80-89 (2014).

41. Hou, D. et al. $130-\mathrm{GHz}$ on-chip meander slot antennas with stacked dielectric resonators in standard CMOS technology. IEEE Trans. Antennas Propag. 60(9), 4102-4109 (2012).

42. Hou, D. et al. Silicon based on-chip antenna design for millimeter-wave/THz application. In Elect. Design of Adv. Pkg. and Syst. Symp (EDAPS), 1-4 (2011).

43. Li, X., Xiao, J., Qi, Z., \& Zhu, H. Broadband and high-gain millimeter-wave and terahertz antenna arrays. In Int. Conference on Microwave and Millimeter Wave Technology (ICMMT), 1-3 (2019).

44. Benakaprasad, B., Eblabla, A., Li, X., Thayne, I., Wallis, D. J., Guiney, I., Humphreys, C. \& Elgaid, K. Terahertz monolithic integrated circuits (TMICs) array antenna technology on GaN-on-low resistivity silicon substrates. In 41st Int. Conference on Infrared, Millimeter, and Terahertz waves (IRMMW-THz), 1-2 (2016).

45. Zhu, H., Li, X., Qi, Z. \& Xiao, J. A $320 \mathrm{GHz}$ octagonal shorted annular ring on-chip antenna array. IEEE Access 8, 84282-84289 (2020).

\section{Acknowledgements}

This work was supported in part by the Ministerio de Ciencia, Innovación y Universidades, Gobierno de España (MCIU/AEI/FEDER, UE) under Grant RTI2018-095499-B-C31, in part by the Innovation Programme under Grant H2020-MSCA-ITN-2016 SECRET-722424, and in part by the U.K. Engineering and Physical Sciences Research Council (EPSRC) under Grant EP/E022936/1. 


\section{Author contributions}

Conceptualization, M.A., B.S.V., S.A., C.H.S., R.A.-A., I.H., F.F., and E.L.; methodology, M.A., B.S.V., S.A., C.H.S., N.S., S.J.F., A.A.A., R.A.-A., I.H., F.F., and E.L.; software, M.A., B.S.V., S.S., C.H.S., R.A.-A., I.H., and J.A.M.; validation, M.A., B.S.V., S.S., S.A., C.H.S., N.S., S.J.F., A.A.A., R.A.-A., I.H., J.A.M., F.F., and E.L.; formal analysis, M.A., B.S.V., S.A., C.H.S., S.J.F., A.A.A., R.A.-A., I.H., J.A.M., F.F., and E.L.; investigation, M.A., B.S.V., S.A., C.H.S., R.A.-A., I.H., J.A.M., F.F., and E.L.; resources, M.A., B.S.V., S.S., S.A., C.H.S., N.S., S.J.F., A.A.A., R.A.-A., I.H., J.A.M., F.F., and E.L.; data curation, M.A., B.S.V., S.S., S.A., C.H.S., N.S., S.J.F., J.A.M., F.F., and E.L.; writing-original draft preparation, M.A.; writing-review and editing, M.A., B.S.V., S.S., S.A., C.H.S., N.S., S.J.F., A.A.A., R.A.-A., I.H., J.A.M., F.F., and E.L.; visualization, M.A., B.S.V., S.A., C.H.S., N.S., R.A.-A., I.H., F.F., and E.L.; supervision, E.L.; project administration, R.A.-A., F.F., and E.L.; funding acquisition, R.A.-A., F.F., and E.L.

\section{Competing interests}

The authors declare no competing interests.

\section{Additional information}

Correspondence and requests for materials should be addressed to M.A.

Reprints and permissions information is available at www.nature.com/reprints.

Publisher's note Springer Nature remains neutral with regard to jurisdictional claims in published maps and institutional affiliations.

(c) (i) Open Access This article is licensed under a Creative Commons Attribution 4.0 International License, which permits use, sharing, adaptation, distribution and reproduction in any medium or format, as long as you give appropriate credit to the original author(s) and the source, provide a link to the Creative Commons licence, and indicate if changes were made. The images or other third party material in this article are included in the article's Creative Commons licence, unless indicated otherwise in a credit line to the material. If material is not included in the article's Creative Commons licence and your intended use is not permitted by statutory regulation or exceeds the permitted use, you will need to obtain permission directly from the copyright holder. To view a copy of this licence, visit http://creativecommons.org/licenses/by/4.0/.

(C) The Author(s) 2021 